\title{
Article
}

\section{Effect of Ashwagandha (Withania Somnifera) Root Extract Against Gentamicin Induced Changes of Serum Urea and Creatinine Levels in Rats}

\author{
Sadia Choudhury Shimmi ${ }^{1}$, Nasim Jahan ${ }^{2}$, Nayma Sultana ${ }^{3}$
}

\begin{abstract}
Background: Kidney is an important excretory organ. Its damage can be occurred due to prolonged use and higher doses of drugs, exposure to some chemicals, toxins, or infectious agents. Herbal plants as Ashwagandha (Withania somnifera) may have free radical scavenging activity thereby can be used for the prevention and treatment of kidney damage. Objective: To observe the nephroprotective effect of Ashwagandha (Withania somnifera) root against gentamicin induced nephrotoxicity in Wistar albino rats. Methods: This experimental study was carried out in the Department of Physiology, Sir Salimullah Medical College (SSMC), Dhaka from 1 ${ }^{\text {st }}$ July 2010 to $30^{\text {th }}$ June 2011. A total number of 35 Wistar albino rats, age ranged from 90 to 120 days, weighing between 150 to 200 grams were included in this study. After acclimatization for 14 days, they were divided into control group (Group A) and experimental group (Group B). Control group was again subdivided into group $\mathrm{A}_{1}$ (baseline control, consisted of 10 rats) and group $\mathrm{A}_{2}$ (gentamicin treated control group, consisted of 10 rats). Again, experimental group (Group B- Ashwagandha pretreated and gentamicin treated group) consisted of 15 rats. All groups of animals received basal diet for 22 consecutive days. In addition to this, group $\mathrm{A}_{2}$ also received gentamicin subcutaneously $\left(100 \mathrm{mg} / \mathrm{kg}\right.$ body weight/day) for the last eight $\left(15^{\text {th }}\right.$ to $22^{\text {nd }}$ day) consecutive days. Again, group B received ashwagandha root extract $(500 \mathrm{mg} / \mathrm{kg}$ body weight/ day; orally) for 22 consecutive days and gentamicin subcutaneously (100 $\mathrm{mg} / \mathrm{kg}$ body weight /day) for last eight $\left(15^{\text {th }}\right.$ to $22^{\text {nd }}$ day $)$ days. All the animals were sacrificed on $23^{\text {rd }}$ day. Then blood and kidney sample were collected. Estimation of serum urea, creatinine levels were done by using standard Laboratory kits. The statistical analysis was done by one way ANOVA and Bonferroni test as applicable. Results: The mean serum urea, creatinine levels were significantly $(\mathrm{p}<0.001)$ higher in gentamicin treated control group in comparison to those of baseline control. Again, these levels were significantly $(\mathrm{p}<0.01)$ lower in ashwagandha pretreated and gentamicin treated group (experimental group) when compared to those of gentamicin treated group (control). Conclusion: Ashwagandha (Withania somnifera) root may have some nephroprotective effect against gentamicin induced nephrotoxicity.
\end{abstract}

Key words: Nephroprotective, Ashwagandha, Gentamicin

J Bangladesh Soc Physiol. 2011 December; 6(2): 84-89

For Authors Affiliation, see end of text.

http://www.banglajol.info/index.php/JBSP

\section{Introduction}

7 he kidney is an essential excretory organ of our body, plays a dominant role in homeostasis by excreting the

Received August 2011; Accepted November 2011 metabolic waste products and excess necessary substances. It conserves necessary products depending on the needs of the body ${ }^{1}$. It is prime target of several drugs, toxic xenobiotics or 
chemicals due to high rate of blood flow (20\%$25 \%$ of cardiac output) and presence of cellular transport systems that causes accumulation of these compounds within the nephron epithelial cells ${ }^{2}$. Metabolites of the drugs that are excreted from kidney may also cause cellular damage leading to kidney dysfunction. Several xenobiotic substances like aminoglycosides, cephalosporins, anticancer drugs (cisplatin), amphotericin B, analgesics etc exert their toxic effects by one or more common pathogenic mechanism that can produce nephrotoxicity ${ }^{3}$.

Kidney disease is one of the commonest cause of hospitalization in most of the countries. Acute renal failure (ARF) is a common and serious renal problem having high morbidity and mortality rate $^{4}$. Increase in the levels of blood urea and creatinine is the principal diagnostic criteria of renal failure. Severe and progressive uremia may result in death ${ }^{5}$.

Gentamicin is a broad-spectrum antibiotic belonging to the amino glycosides group, very effective against gram-negative and some grampositive bacterial infections ${ }^{6}$. Gentamicin is well recognized, to produce renal tubular necrosis mainly in the proximal tubule. This drug causes generation of reactive oxygen species (ROS), which induces cell injury and necrosis via lipid peroxidation ${ }^{7.8}$.

Withania somnifera, known as ashwagandha / Winter cherry/ Indian ginseng, widely used in the Ayurvedic medicine, belongs to the family of Solanaceae. Leaves, fruits, seeds, shoots and roots of this plant have all been used traditionally as well ${ }^{9}$. The roots of Withania somnifera contained 35 chemical constituents. Withaferin $\mathrm{A}$ and withanolides, the active ingredients contribute to the most of the biological actions of Withania. Furthermore, the roots of this plant are reputed to promote health and longevity by augmenting defense against some diseases, arresting the aging process, revitalizing the body in debilitated condition, increasing the capability of individual to resist adverse environment factors and by creating a sense of mental well being ${ }^{10}$. Till today, no side effects have been found in ashwagandha ${ }^{11}$.
Some researchers observed that Withania somnifera significantly reduced gentamicin induced elevation of serum urea and creatinine levels in rats ${ }^{12}$. Recently some researchers showed that the root of this plant lowers the dehydration-induced increase in the level of serum urea and creatinine indicates protective effect of this plant against renal injury ${ }^{11}$. Moreover, some other researchers found that root of Withania somnifera significantly inhibit lipid peroxidation and generation of free radicals to provide protection against nephrotoxicity ${ }^{13}$, 14. Whereas, some other researchers found ashwagandha (Withania somnifera) induced marked renal lesions in rats ${ }^{15}$.

Now a days, there is increasing incidence of renal failure with consequence of high morbidity and mortality in our country. So, preventions of occurrence and progression of acute renal failure (ARF) has become a very important issue. But modern medicine lacks of reliable nephroprotective drugs. In recent years, great effort has been focused on traditional and herbal medicine for the treatment of $\mathrm{ARF}^{16}$. But in Bangladesh, little is known about the nephroprotective effect of ashwagandha (Withania somnifera).Therefore, the present study has been designed to observe the nephroprotective role of ashwagandha (Withania somnifera) in experimental animals after inducing nephrotoxicity by gentamicin. It is also expected that the result of this study would make the ashwagandha acceptable among the people as a rich source with medicinal value for the prevention of kidney diseases.

\section{Methods}

This experimental study was conducted between July 2010 to June 2011 in the Department of Physiology, SSMC, Mitford, Dhaka. A total number of 35 Wistar albino rats, age ranged from 90 to 120 days, weighing between 150 to 200 grams were included in this study. After acclimatization for 14 days, they were divided into control group (Group A) and experimental group (Group B). Control group was again subdivided into group $\mathrm{A}_{1}$ (baseline control, consisted of 10 rats) and group $A_{2}$ (gentamicin 


\section{Article}

treated control group, consisted of 10 rats). Again, experimental group (Group BAshwagandha pretreated and gentamicin treated group) consisted of 15 rats. All groups of animals received basal diet for 22 consecutive days. In addition to this, group $\mathrm{A}_{2}$ (gentamicin treated group) also received gentamicin subcutaneously (100mg $/ \mathrm{kg}$ body weight/day) for the last eight ( $15^{\text {th }}$ to $22^{\text {nd }}$ day) consecutive days. Again, group B (ashwagandha pretreated and gentamicin treated group) received ashwagandha root extract $(500 \mathrm{mg} / \mathrm{kg}$ body weight/day; orally) for 22 consecutive days and gentamicin subcutaneously $(100 \mathrm{mg} / \mathrm{kg}$ body weight /day) for last eight ( $15^{\text {th }}$ to $22^{\text {nd }}$ day) days. After giving gentamicin and ashwagandha all the animals including baseline control rats, were anaesthetized with the help of chloroform and sacrificed on $23^{\text {rd }}$ day. After 14 days of acclimatization, body weights were measured (initial bw), Again, final body weights (final bw) of rats before anaesthetized on $23^{\text {rd }}$ day were taken. The blood and kidney samples were collected. Kidney was washed in ice cold saline. Then, it was wiped in tissue paper and weighed by electric balance analyzer. Blood was centrifuged at the rate of $4000 \mathrm{rpm}$ for 5 minutes and supernatant serum was collected. Out of 35
Effect of Ashwagandha on serum urea and creatinine levels

serum samples, four (4) were hemolyzed. So, thirty one (31) samples were estimated. Assessment of kidney function was done by estimating serum urea (modified urease-berthelot colorimetric method in auto analyzer ${ }^{17}$ )and creatinine (alkaline picrate method in auto analyzer ${ }^{18}$ ) levels in the laboratory of Department of Physiology, SSMC. Statistical analysis were done by one way ANOVA and Bonferroni test by using SPSS windows, version 16.

Preparation of root extract of ashwagandha (Withania somnifera):

Ashwagandha is cultivated and harvested in the Ayurvedic nursery of Hamdard

Laboratories, Meghna. After collecting the roots of this plant, it was dried in sunlight for 2 days, crushed in an electrical grinder into powder. Then the powder was extracted in methanol, filtered, evaporated by rotatory evaporator and dried. After that, the dried root extract of ashwagandha was dissolved by propylene glycol $(2 \mathrm{ml} / \mathrm{kg}$ body weight) and finally mixed with distilled water for feeding.

\section{Results}

Body weights of the rats are presented in Table I.

Table I: Initial and final body weight with the percent of change of body weight in different groups ( $\mathrm{n}=31)$

\begin{tabular}{|c|c|c|c|}
\hline \multirow[t]{2}{*}{ Groups } & \multicolumn{2}{|c|}{ Body weight (g) } & \multirow{2}{*}{ initial (I) weight $[(\mathrm{F}-\mathrm{I}) / \mathrm{I} \times 100]$} \\
\hline & Initial (Day 1) & Final (Day 23) & \\
\hline$\overline{A_{1}(n=9)}$ & $181.13 \pm 2.64$ & $187.18 \pm 3.66$ & $3.34 \pm 0.92$ \\
\hline$A_{2}(n=9)$ & $182.30 \pm 2.80$ & $176.83 \pm 3.69$ & $-2.99 \pm 1.47$ \\
\hline $\mathrm{B}(\mathrm{n}=13)$ & $181.85 \pm 3.13$ & $180.46 \pm 2.59$ & $-0.75 \pm 0.79$ \\
\hline \multicolumn{4}{|c|}{ Statistical analysis } \\
\hline \multicolumn{4}{|c|}{$P$ value } \\
\hline$\overline{A_{1}}$ vs $A_{2}$ & $0.189^{\text {ns }}$ & $0.000^{* * * * *}$ & $0.001^{* *}$ \\
\hline$A_{1}$ vs $B$ & $0.285^{\mathrm{ns}}$ & $0.000^{* * *}$ & $0.004^{* *}$ \\
\hline $\mathrm{A}_{2}$ vs $\mathrm{B}$ & $0.363^{\mathrm{ns}}$ & $0.012^{*}$ & $0.003^{* *}$ \\
\hline
\end{tabular}

Results are expressed as Mean \pm SD. Statistical analysis was done by ANOVA test and then Bonferroni test was performed to compare between groups.

Group $A_{1}$ (Baseline control group) Group $A_{2}$ (Gentamicin treated control group)

Group B (Ashwagandha pretreated and gentamicin treated group)

ns: Non-significant $*$ : Significant at $\mathrm{p}<0.05 * *$ : Significant at $\mathrm{p}<0.01$

$* * *$ : Significant at $\mathrm{p}<0.001$ 
The kidney weight was significantly $(\mathrm{p}<0.01)$ higher in group $A_{2}$ when compared to those of group $A_{1}$ and $B$. Again, kidney weight of group $B$ was higher than that of group $A_{1}$, but the difference was not statistically significant (Figure 1).

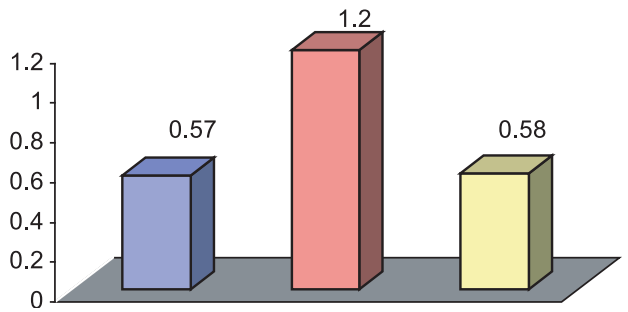

Figure 1: Mean kidney weight of different groups of rats $(n=31)$

Group $\mathrm{A}_{1}$ (Baseline control group)

Group $A_{2}$ (Gentamicin treated control group)

Group B (Ashwagandha pretreated and gentamicin treated group)

Table II: Serum urea and creatinine levels in different groups of rats $(\mathrm{n}=31)$

\begin{tabular}{lcc}
\hline Groups & $\begin{array}{c}\text { Serum urea } \\
(\mathrm{mg} / \mathrm{dl})\end{array}$ & $\begin{array}{c}\text { Serum Creatinine } \\
(\mathrm{mg} / \mathrm{dl})\end{array}$ \\
\hline $\mathrm{A}_{1}(\mathrm{n}=9)$ & $50.78 \pm 2.05$ & $0.88 \pm 0.18$ \\
$\mathrm{~A}_{2}(\mathrm{n}=9)$ & $127.56 \pm 4.71$ & $2.70 \pm 0.25$ \\
$\mathrm{~B}(\mathrm{n}=13)$ & $63.23 \pm 1.48$ & $1.13 \pm 0.13$ \\
\hline \multicolumn{3}{c}{ P value } \\
\hline $\mathrm{A}_{1}$ vs $\mathrm{A}_{2}$ & $0.000^{* * *}$ & $0.000^{* * *}$ \\
$\mathrm{~A}_{1}$ vs B & $0.017^{*}$ & $0.025^{* *}$ \\
$\mathrm{~A}_{2}$ vs B & $0.004^{* *}$ & $0.007^{* *}$
\end{tabular}

Results are expressed as Mean \pm SD. Statistical analysis was done by ANOVA test and then Bonferroni. Group $A_{1}$ (Baseline control group) Group $A_{2}$ (Gentamicin treated control group)

Group B (Ashwagandha pretreated and gentamicin treated group), $\mathrm{n}:$ Number of subjects

$*$ : Significant at $\mathrm{p}<0.05 * *$ : Significant at $\mathrm{p}<0.01$

$* * *$ : Significant at $\mathrm{p}<0.001$

The mean serum levels of urea and creatinine were significantly higher in group $\mathrm{A}_{2}(\mathrm{p}<0.001)$ and group $B(p<0.05)$ in comparison to those of group $A_{1}$. Again, these levels of group B were significantly $(\mathrm{p}<0.01)$ lower than those of group $\mathrm{A}_{2 \text {. }}$ (Table II).

\section{Discussion}

In this study, the kidney weight was significantly higher in gentamicin treated control rats when compared to that of baseline control and experimental group. But, no significant difference of this value was observed between experimental group and baseline control group. This finding is in consistent with that of some other investigators $^{12,19}$.

In the present study, serum levels of urea and creatinine were significantly higher in gentamicin treated control and in experimental group in comparison to those of baseline control group. Again, significantly lower levels of these parameters were observed in experimental group when compared to those of gentamicin treated control group. Similar findings were also reported by different researchers 11,20 .

It has been suggested that, toxic dose of gentamicin may decrease the plasma magnesium concentration. This hypomagnesaemia may reduce the body weight, as magnesium is an essential mineral required for regulation of carbohydrate, protein and lipid metabolism and for maintenance of normal health ${ }^{21}$.

It has been postulated that, gentamicin induced toxicity may be produced by accumulation and dumping of gentamicin in kidney which causes oedema and inflammatory changes in the proximal tubular epithelial cells and thereby increases the kidney weight ${ }^{22,23}$.

Again, toxic dose of gentamicin causes impairment of renal microcirculation and glomerular hemodynamics thus, decreases glomerular filtration rate (GFR) and thereby increases serum urea and creatinine levels ${ }^{7,23}$. However, gentamicin induced toxicity may cause excessive formation of ROS (reactive oxygen species) such as $\mathrm{O}_{2}{ }^{-}$, $\mathrm{OH}^{-}$and $\mathrm{H}_{2} \mathrm{O}_{2}$ that stimulate mesangial cells contraction. So, there is decreased GFR (glomerular filtration rate) and increased serum urea and creatinine levels ${ }^{12,24}$.

However, some other researchers suggested that, ashwagandha root contained sitoindosides VII- 


\section{Article}

$\mathrm{X}$ and withaferin $\mathrm{A}$, have antioxidant activity by enhancing the free radical scavenging enzymes such as, superoxide dismutase (SOD), catalase (CAT), glutathione peroxidase $(\mathrm{GPx})^{9,} 10$. Therefore, it may suppress toxin-mediated enhancement of serum urea and creatinine levels and improvement of body weight ${ }^{11,12}$.

Furthermore, ashwagandha also have the ability to decrease serum urea and creatinine levels by increasing glomerular filtration rate may be due to some of its active components like phenolic compounds and flavonoids ${ }^{14}$.

Moreover, it has been suggested that ashwagandha root extract contained withanolides, which inhibit cyclooxygenase enzymes, lipid peroxidation and proliferation of tumor cells. Thus, it reduces inflammatory process by inactivating nuclear factor -kappa B $\left(\mathrm{NF}-{ }^{\circ} \mathrm{B}\right)$ activation. This anti-inflammatory property may be helpful in decreasing the kidney weight ${ }^{25}$.

In the present study, nephrotoxicity was observed in rats treated with gentamicin as evidenced by higher levels of serum urea and creatinine. This is further supported by an increase in kidney weight in nephrotoxic rats of present study which may be due to increased production of free radicals and this may initiate lipid peroxidation and subsequent cellular damage.

Again, lower levels of serum urea and creatinine and decrease in kidney weight in ashwagandha pretreated and gentamicin treated rats of the present study suggested the nephroprotective role of this root extract against gentamicin toxicity. These effects are most likely due to free radical scavenging activity of ashwagandha. However, the exact mechanism involved in the nephroprotective activity of ashwagandha (Withania somnifera) root extract against gentamicin induced nephrotoxicity in rats cannot be find out from this type of study.
Effect of Ashwagandha on serum urea and creatinine levels

\section{Conclusion}

From this study, it can be concluded that ashwagandha (Withania somnifera) root extract may have some role in decreasing serum urea and creatinine levels and normalize the kidney weight against gentamicin toxicity, which may be due to its inhibition of generating and scavenging free radicals.

\section{Acknowledgement}

Authors of this study are thankful to the authority of Pharmacy Department of Dhaka University, Hamdard Laboratories and BCSIR, Dhaka for the cooperation they provided. We would also express our sincere thanks to the DGHS for providing the partial financial support for completing the research work.

\section{Authors Affiliation}

1. * Sadia Choudhury Shimmi, Lecturer, Department of Physiology, Delta Medical College and Hospital, Dhaka. E-mail:shimmi_cmc40@yahoo.com

2. Nasim Jahan, Professor and Head, Department of Physiology, Sir Salimullah Medical College, Dhaka. E-mail: prof.dr.nasimjahan@gmail.com

3. Nayma Sultana, Associate Professor, Department of Physiology, Sir Salimullah Medical College, Dhaka. Email : nayma_sultana@yahoo.com

* for correspondence

\section{References}

1. Hall JE. Text Book of Medical Physiology. $12^{\text {th }}$ ed. Philadelphia: Saunders Elsevier; 2011.p 307326.

2. Pfaller W, Gstraunthaler G. Nephrotoxicity testing in vitro- what we know and what we need to know. Environ Health perspect. 1998; 106(2): 559-569.

3. Naughton C.A. Drug induced nephrotoxicity. Am Fam Physician 2008; 78 (6): 743-750.

4. Begum NA, Dewan ZF, Nahar N, Mamun MIR. Effect of n-Hexane extract of Nigella sativa on gentamicin induced nephrotoxicity in rats. Bangladesh J Pharmacol 2006; 1: 16-20.

5. Anderson WAD, Jones DB. Kidneys. In: Anderson WAD (ed.), Pathology, $6^{\text {th }}$ ed, The C.V Mosby Company; 1971. p772-776 . 
6. Gingell JC, Water worth PM. Dose of gentamicin in patients with normal renal function and renal impairment. Brit. Med.J. 1968; 2: 19-22.

7. Stojiljkovic N, Veljkovic S, Mihailovic D, Stoiljkovic M, Radovanovic D, Randelovic P. The effect of calcium channel blocker Verapamil on gentamicin nephrotoxicity in rats. Bosn J Basic Med Sci 2008; 8(2): 170-176.

8. Silan C, Uzun O, Comunoglu NU, Gokcen S, Bedirhan S, Cengiz M. Gentamicin-induced nephrotoxicity in rats ameliorated and healing effects of Resveratrol. Biol. Pharm. Bull. 2007; 30(3): 79-83.

9. Mishra LC. Scientific Basis for the therapeutic use of Withania somnifera (Ashwagandha): A Review. Altern Med Rev.2000; 5(4): 334-346.

10. Bhattacharya A, Ghosal S, Bhattacharya SK. Antioxidant effect of Withania somnifera glycowithanolides in chronic foot shock stressinduced perturbations of oxidative free radical scavenging enzymes and lipid peroxidation in rat frontal cortex and striatum. J Ethnopharmacol. 2010; 74(1): 1-6.

11. Das K, Samanta TT, Samanta P, Nandi DK. Effect of extract of Withania somnifera on dehydration -induced oxidative stress- related uremia in male rats. Saudi J of Kidney dis Transpl 2010; 21(1): 7580 .

12. Jeyanthi T, Subramanian P. Nephroprotective effect of Withania somnifera: A dose- dependent study. Renal failure 2009; 31(9): 814-821.

13. Jeyanthi T, Subramanian P. Protective effect of Withania somnifera root powder on lipid peroxidation and antioxidant status in gentamicin -induced nephrotoxic rats. J Basic Clin Physiol Pharmacol 2010; 21(1): .61-78.

14. Harikrishnan B, Subramanian P, Subash S. Effect of Withania somnifera root powder on the levels of circulatory lipid peroxidation and liver marker enzymes in chronic Hyperammonemia. E.Journal of Chemistry 2008; 5(4): 872-877.

15. Khan S, Balick M.J. Therapeutic plants of Ayurveda: A review of selected Clinical and other studies for
166 species. The Journal of Alternative and Complementary Medicine 2001; 7(5): 405-515.

16. Zhang HA, Wang M, Zhou J, Yao QY, Ma JM, Jiang, CL. Protective effect of Ginsenoside against acute renal failure and expression of tyrosine hydroxylase in the locus coeruleus. Physio.Res 2010; 59: 61-70.

17. Fawcett JK, Scott JE. J. Cin. Path 1960; 13: .156159.

18. Bartels H, Bohmer M. Clin. Chem. Acta 1972; 37: 193.

19. Erdem A, Gundogan NU, Usubutun A, Kilinc K, Erdem SR, Kara A, Bozkurt A. The protective effect of taurine against gentamicin-induced acute tubular necrosis in rats. Nephrol Dial Transplant 2010; 15(8): 1175-1182.

20. Borde AU, Qureshi MI, Patil MK, Mendhe MS, Athawale AM. Protective effect of Withania Somnifera on cadmium chloride induced hematological and biochemical changes in male rats. J Res Educ Indian Med 2008; 15(2): 15-20.

21. Whang R. Magnesium deficiency, causes and clinical implications. Drugs 1984; 1: 143-150.

22. Yasin KF, Sabir M, Sherwani MIK, Zafar M, Yasmin S, Alam MI. Amelioration of gentamicin nephrotoxicity by vitamin B6 (a general and biochemical profile). Pakistan JMed Res 2003; 42(2): 69-73.

23. Patil CR, Jadhav RB, Singh PK, Mundada S, Patil PR. Protective effect of Oleanolic Acid on Gentamicin Induced Nephrotoxicity in Rats. Phytother Res 2010; 24: 33-37.

24. Maldonado PD, Berrera D, Compos ONM, Pando RH, Rubio MEI, Chaverri JP .Aged garlic extract attenuates gentamicin induced renal damage and oxidative stress in rats. Life Sci 2003; 73(20): 2543-2556.

25. Ichikawa $\mathrm{H}$, Takada $\mathrm{Y}$, Shishodia $\mathrm{S}$, Jayaprakasham B, Nair MG, Aggarwal BB. Withanolides potentiate apoptosis, inhibits invasion and abolish osteoclastogenesis through suppression of nuclear factor-kappa B (NF- ${ }^{\circ}$ ) activation and NF-kappa B- regulated gene expression. Mol Cancer Ther 2006; 5(6): 1434-1445. 